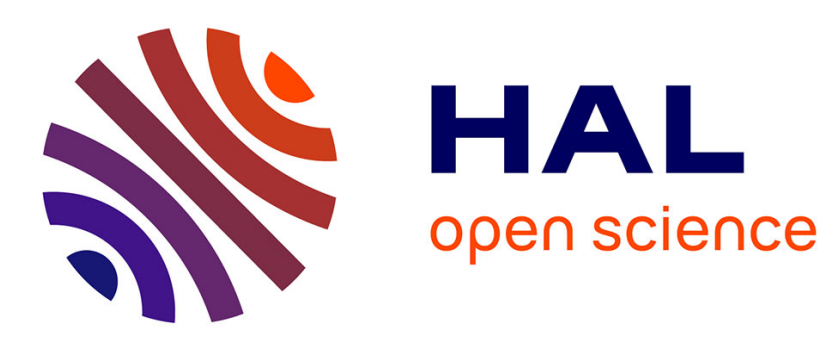

\title{
Effect of Circular Arc Feet on a Control Law for a Biped
} Tetsuya Kinugasa, Christine Chevallereau, Yannick Aoustin

\section{To cite this version:}

Tetsuya Kinugasa, Christine Chevallereau, Yannick Aoustin. Effect of Circular Arc Feet on a Control Law for a Biped. Robotica, 2009, 27, pp.621-632. 10.1017/S0263574708005006 . hal-00794784

\section{HAL Id: hal-00794784 \\ https://hal.science/hal-00794784}

Submitted on 26 Feb 2013

HAL is a multi-disciplinary open access archive for the deposit and dissemination of scientific research documents, whether they are published or not. The documents may come from teaching and research institutions in France or abroad, or from public or private research centers.
L'archive ouverte pluridisciplinaire HAL, est destinée au dépôt et à la diffusion de documents scientifiques de niveau recherche, publiés ou non, émanant des établissements d'enseignement et de recherche français ou étrangers, des laboratoires publics ou privés. 


\title{
Effect of Circular Arc Feet on a Control Law for a Biped
}

\author{
Tetsuya Kinugasa, Christine Chevallereau and Yannick Aoustin \\ IRCCyN (Institut de Recherche en Communications et en Cybernétique de Nantes) \\ 1 rue de la Noë BP 92101, 44321 Nantes Cedex 3, France \\ Email: \{Tetsuya.Kinugasa, Christine.Chevallereau, Yannick.Aoustin\}@irccyn.ec-nantes.fr
}

\begin{abstract}
The purpose of our research is to study the effects of circular arc feet on the biped walk with a geometric tracking control. The biped studied is planar and is composed of five links and four actuators located at each hip and each knee thus the biped is underactuated in single support phase. A geometric evolution of the biped configuration is controlled, instead of a temporal evolution. The input-output linearization with a PD control law and a feed forward compensation is used for geometric tracking. The controller virtually constrains four degrees of freedom (DoF) of the biped, and one DoF (the absolute orientation of the biped) remained. The temporal evolution of the remained system with impact events is analyzed using Poincaré map. The map is given by an analytic expression based on the angular momentum about the contact point. The effect of the radii of the circular arc feet on the stability is studied. As a result, the speed of convergence decreases when the radii increases, if the radius is larger than the leg length the cyclic motion is not more stable. Among the stable cyclic motion, choosing larger radius broadens the basin of attraction. Our results agree with those obtained for passive dynamic walking on stability, even if the biped is controlled through the geometric tracking.
\end{abstract}

\section{INTRODUCTION}

Over the past several years a considerable amount of studies have been proposed on biped walking. The choice of type of feet such as a contact points, flat feet and circular arc feet is important, because walking stability is essentially affected by the contact with the ground. Control methods of many traditional humanoids with flat foot are based on zero moment point (ZMP) that remains inside the convex hull of the foot support using the ankle torque. There are lots of successful results, but the gaits seem not to be so natural. On the other hand, for a biped with point contact a geometric tracking method for biped walking using input-output linearization (e.g. [1]-[4]) produces stable gait that seems quite natural (the geometric tracking method was originally proposed by Kajita [5]). Grizzle et. al. [2] proposed the method for a threelink model, only two outputs are controlled, the reference are expressed as a function of the biped state. Zero dynamics with an impact event of the controlled system were analyzed by Poincaré method. The effectiveness of geometric tracking has been verified on a platform called 'Rabbit' [4] (Fig.1 left) with point feet. Westervelt et. al. [6] gave some additional results to show capability for robustness, changing average walking rate, and rejecting a perturbation by 'one-step transition control' and 'event-based control'.

In the domain of passive dynamic walking mechanisms [11], it is shown that a biped with large radius circular arc feet can take easily a lot of steps. The prototype Emu (Fig.1 right) can be equipped with various arc feet with different radii ([7] and [8]). In previous walking experiments the biped Emu is excited by gravity or forced oscillation of the length of legs. If the feet radius is $10 \%$ of leg length, the biped could only take few steps [7] excited by the effect of gravity because of the sensitivity to disturbances produced by the cables, the guide to avoid lateral motion and so on. The biped could not walk by the forced oscillation. In the case of a radius which is $97 \%$ of leg length, the biped Emu (Fig.1 right) can take easily few dozen of steps [8] by the gravity and the leg oscillations. The step number is limited only by the space of our laboratory. The effect of the radii of circular feet was significant for our results, but the change of radius is also accompanied by other difference in physical parameters, thus a direct conclusion on the experimental study is not obvious and a more rigorous study must be done. In fact, the same results are well known in the field of passive dynamic walking as it is mentioned in Section II.

The geometric tracking method that was used for the underactuated biped Rabbit can be extended to the case of underactuated biped with circular arc feet. If the biped has the circular arc feet, the analytical stability study given in [4] can not be applied directly. The contact point between the supporting foot and the ground moves forward during the step in this case. The same difficulty appears also in a flat feet model. For this problem, Djoudi and Chevallereau [9] gave a solution to analyze the stability with a chosen evolution of the ZMP.

The purpose of the paper is to show the effects of the circular arc feet for an underactuated planar biped controlled by a geometric tracking method. The effect of the feet shape on the control properties is obviously depending on the walking strategies. Therefore it is significant to clarify the effect of the feet shape on the geometric tracking even if it is well know in the passive dynamic walking field.

A model of our biped is composed of five links. Prismatic knee joints are employed to avoid the foot clearance problem which occurs in association with large foot, not actuated ankle and rotational knee joint. A geometric evolution of the biped configuration is controlled, instead of a temporal evolution. The input-output linearization with a PD control law and a feed forward compensation is used for geometric tracking. 

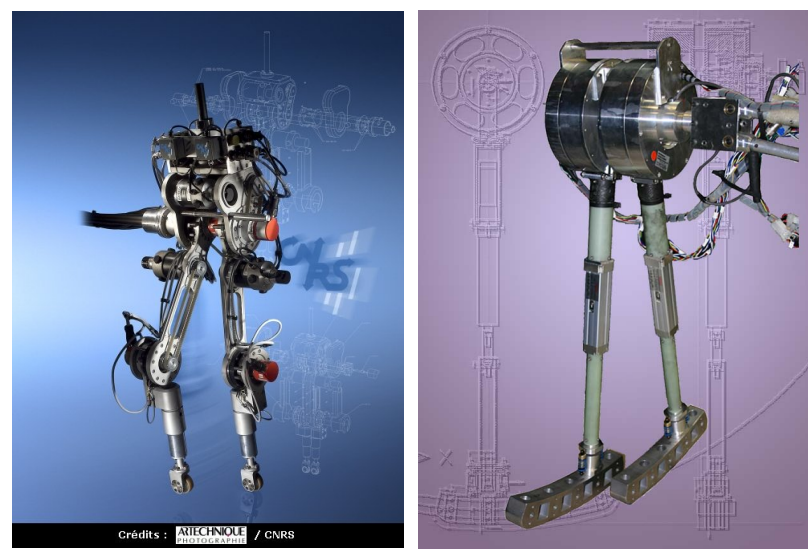

Fig. 1. Biped bipeds, "Rabbit" (left) and "Emu" (right).

The temporal evolution is analyzed using Poincaré map. The map is given by an analytic expression based on the angular momentum about the mobile contact point. The effect of the radius of the circular arc feet on stability and the basin of attraction is revealed by analytic calculation. It is compared to the effect of radius of the circular arc feet on passive dynamic walking. Section II presents an overview of previous studies on the circular arc feet. Section III gives the biped model. It is composed of a dynamic model and the impact model (instantaneous double support). Section IV presents the control method. Section V gives the stability analysis. Some simulation results are shown in Section VI and some discussion on the effects of the feet radius is developed in Section VI-E. Section VII concludes the paper.

\section{Previous studies on Biped with Circular ARC FEET}

A circular arc feet for the biped are often treated in the field of passive dynamic walking [11]. It is well known that a passive dynamic walking gives an extremely natural gait. McGeer showed that an eigenvalue of the "speed mode" came to unit when the radius of a circular arc foot approaches the length of legs, and the eigenvalue becomes unit for synthetic wheel which has the foot radius equals to the leg length. The "speed mode" was related to dissipation of energy at the impact.

Wisse et. al. [12] showed that the larger feet radius, the larger amount of disturbances is accepted in experiments. The robustness against disturbances is connected to the size of a basin of attraction for walking. Wisse explained in [13] that "The walker will fall backward if it has not enough velocity to overcome the vertical position. Circular feet smoothen the hip trajectory and thus relax the initial velocity requirement. As the result, the basin of attraction is enlarged." However a decisive study on the effect of circular arc feet on the basin of attraction has yet to be performed. Recently, Wisse et. al. [14] presented a stability analysis of passive dynamic walking with flat feet and passive ankles. The effect of the flat feet was analogous to the effect of the circular arc feet for many properties in the sense that ZMP smoothly and monotonically moves forward from heel to toe. However he pointed out the need of validation for a more accurate model of the heel strike transition. Asano and Luo [15] discussed similar effect between the circular arc feet and the flat feet with actuated ankles.

Adamczy, Collins and Kuo [16] studied the centre of mass (CoM) mechanical work per step with respect to foot radius for various simple models of biped powered by an instantaneous push-off impulse under the stance foot just before contralateral heel strike [18]. They also showed relationships between foot radius and metabolic costs from measured via respiratory gas exchange. The data are collected through human walking with feet attached to rigid arc, and they conclude that the most effective walking is obtained when the foot radius equals to $30 \%$ of leg length. Geometrically speaking, feet length should be at least twice of the product of the coxa angle between two legs and the radius of feet [11].Therefore one might choose the radius as $1 / 3$ of a leg length with an angle 0.3 rad between two legs, in order to make an anthropomorphic biped, as McGeer wrote.

Thus for anthropomorphic models, $1 / 3$ of leg length seems to be desirable in the sense of geometry between step length and feet lengths [11], "foot clearance problem" [12] and energy costs [16].

\section{THE BIPED MOdeling}

A biped presented in Fig.2 is composed of a torso and two symmetric legs which consist of the prismatic frictionless knees and the circular arc feet. The hips are rotational frictionless joints. We assume that the contact point does not slip and the biped walks in a vertical sagittal plane. The vector $\theta=\left[l_{1}, l_{2}, \theta_{1}, \theta_{2}, \theta_{3}\right]^{\prime}$ (" ' " means transpose) of configuration variables (see Fig. 2) describes the shape of the biped during single support, $l_{i}$ is the length of leg $i, \theta_{i}, i=1,2$ is the angle between the torso and the leg $i, \theta_{3}$ is the absolute angle of the supporting leg. The contact point between the biped and the ground is $N_{1}$. The lowest point of the swing leg tip is noted $N_{2}$. The actuator torques and forces are expressed by a vector $\Gamma=\left[\Gamma_{1}, \Gamma_{2}, \Gamma_{3}, \Gamma_{4}\right]^{\prime}$. The absolute orientation of the biped $\theta_{3}$ is not directly actuated. Thus, in a single support (SS), the biped is an under-actuated system. The walking gait consists of single support phases separated by impacts, which are instantaneous double supports where a leg exchange takes place.

\section{A. Dynamic Model for Single Support Phase}

The dynamic model can be written as follows:

$$
D(\theta) \ddot{\theta}+H(\theta, \dot{\theta})=B \Gamma,
$$

where $D \in \Re^{5 \times 5}$ is the inertia matrix, the vector $H \in \Re^{5}$ contains Coriolis, centrifugal and gravity terms. $B \in \Re^{5 \times 4}$ defines how the inputs $\Gamma$ enter the model. Due to the choice of joint coordinates, the matrix $B$ is written as:

$$
B=\left[\begin{array}{c}
I_{4} \\
O_{1 \times 4}
\end{array}\right]
$$




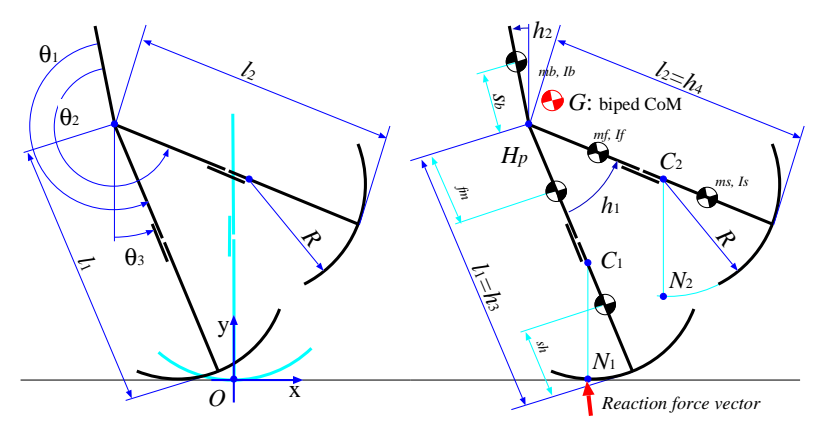

Fig. 2. The biped model

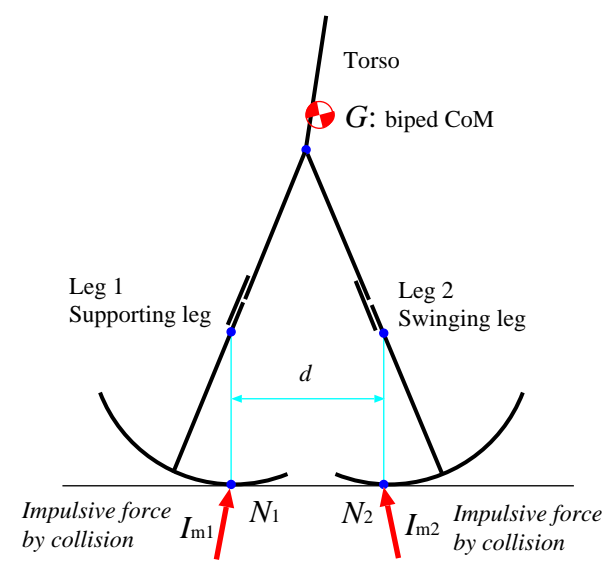

Fig. 3. The biped model at the impact

\section{B. Impact model}

To derive an impact model, an general dynamic model is written:

$$
D_{e}(\theta) \ddot{\theta}_{e}+H_{e}\left(\theta_{e}, \dot{\theta}\right)=B_{e} \Gamma+D_{R_{i}}(\theta) R_{i} .
$$

where $\theta_{e}=\left[\theta^{\prime}, x_{H}, y_{H}\right]^{\prime}$, and $x_{H}$ and $y_{H}$ are the Cartesian coordinates of the hip position $H_{p}$ shown in Fig. $2, D_{e} \in \Re^{7 \times 7}$ is the inertia matrix, the vector $H_{e} \in \Re^{7}$ contains Coriolis, centrifugal and gravity terms. $R_{i}=\left[R_{x_{i}}, R_{y_{i}}\right]^{\prime}$ is a ground reaction force vector applied at the contact point. $B_{e} \in \Re^{7 \times 4}$ and $D_{R_{i}} \in \Re^{7 \times 2}$ defines how the inputs $\Gamma$ and $R_{i}$ enter the model, $i$ is the number of the leg in contact with the ground, $i=1, i=2$, or $i=1,2$.

When the leg $i$ rolls on the ground, the contact with the ground occurs in $N_{i}$. If leg $i$ the ground and since, we assume that no sliding occurs, the position of $N_{i}$ is $O N_{i}=$ $\left[-R \theta_{3}, 0\right]^{\prime}$, where $O$ is defined such that for the current step, the point contact is in 0 when $\theta_{3}$ is zero. This position can also be calculated by : $O N_{i}=O H_{p}+H_{p} C_{i}+C_{i} N_{i}$. Thus, we have :

$$
\left[\begin{array}{c}
-R \theta_{3} \\
0
\end{array}\right]=\left[\begin{array}{c}
x_{H}+\left(l_{i}-R\right) \sin \theta_{3} \\
y_{H}-\left(l_{i}-R\right) \cos \theta_{3}-R
\end{array}\right] .
$$

Therefore, the following constraint equation is obtained:

$$
\Psi_{i}:=\left[\begin{array}{l}
x_{H}+R \theta_{3}+\left(l_{i}-R\right) \sin \theta_{3} \\
y_{H}-R-\left(l_{i}-R\right) \cos \theta_{3}
\end{array}\right]=0 .
$$

Equation (4) is differentiated twice with respect to time, to obtain a constraint on the joint acceleration:

$$
D_{R_{i}}^{\prime} \ddot{\theta}_{e}+C_{R_{i}}\left(\theta_{e}, \dot{\theta}_{e}\right) \dot{\theta}_{e}=0
$$

where $D_{R_{i}}^{\prime}=\partial \Psi_{i} / \partial \theta_{e}$ and $C_{R_{i}}$ comes from the derivation.

We assume that the impact is inelastic and instantaneous without sliding. Let $\dot{\theta}_{e}^{-}$and $\dot{\theta}_{e}^{+}$be the angular velocities just before and just after the impact, respectively. Let $I_{m_{i}}=$ $\left[I_{m x_{i}}, I_{m y_{i}}\right]^{\prime}$, for $i=1,2$ be the vector of magnitudes of the impulsive reaction at the contact point of the stance and the swing leg. During the impact, the previous supporting leg can stay on the ground or take-off. If the leg takes-off, the velocity of $N_{1}$ after the impact is positive. The impulsive ground reaction associated to a leg that stays on the ground must be positive and be in the friction cone. If the supporting leg takes off, the associated impulsive ground reaction is zero. The impact occurs when the leg tip of the swing leg contacts to the ground. To take into account the two cases, the following impact equation can be written:

$$
\left\{\begin{array}{l}
D_{e}(\theta)\left(\dot{\theta}_{e}^{+}-\dot{\theta}_{e}^{-}\right)=D_{R}(\theta) I_{m} \\
D_{R}^{\prime}(\theta) \dot{\theta}_{e}^{+}=0
\end{array},\right.
$$

where,

$$
\begin{aligned}
& D_{R}(\theta)=\left\{\begin{array}{ll}
D_{R_{2}}(\theta), & \dot{y}_{N_{1}}^{+}>0 \\
D_{R_{12}}(\theta), & I_{m y_{1}}>0, I_{m y_{2}}>0
\end{array},\right. \\
& I_{m}=\left\{\begin{array}{ll}
I_{m_{2}}, & \dot{y}_{N_{1}}^{+}>0 \\
I_{m_{12}}, & I_{m y_{1}}>0, I_{m y_{2}}>0
\end{array},\right. \\
& D_{R_{12}}(\theta)=\left[\begin{array}{cc}
D_{R_{1}}(\theta) & 0 \\
0 & D_{R_{2}}(\theta)
\end{array}\right], I_{m_{12}}=\left[\begin{array}{c}
I_{m_{1}} \\
I_{m_{2}}
\end{array}\right] \text {. }
\end{aligned}
$$

From equation (6), we obtain:

$$
\dot{\theta}_{e}^{+}=\left(I_{7 \times 7}-D_{e}^{-1} D_{R}\left(D_{R}^{\prime} D_{e}^{-1} D_{R}\right)^{-1} D_{R}^{\prime}\right) \cdot \dot{\theta}_{e}^{-} .
$$

Before and after the impact, the biped is in contact with the ground on at least one leg, thus $x_{H}, y_{H}$ can be calculated as function of $\theta$, and $\dot{x}_{H}, \dot{y}_{H}$ can be calculated as function of $\dot{\theta}$. Equation (7) can be transformed into an equation of $\theta, \dot{\theta}$ only.

$$
\dot{\theta}^{+}=\Delta(\theta) \dot{\theta}^{-}
$$

where $\Delta(\theta) \in \Re^{5 \times 5}$ is the impact matrix. This matrix depends on the foot radius $R$. In the gait studied, the legs swap their roles from one step to the next, thus since the biped is symmetric, the dynamic model is derived only for the support on leg 1. And the leg exchange is taken into account just after the impact. The state of the biped to begin the next step is :

$$
\theta_{i}=T_{L S} \theta_{f}, \quad \dot{\theta}_{i}=T_{L S} \dot{\theta}^{+}
$$

where $T_{L S} \in \Re^{5 \times 5}$ is the permutation matrix describing the leg exchange, the indexes ${ }_{i}, f$ denoted the initial and final states of the biped for one step. 


\section{Control law}

Since the studied biped is underactuated, and since some good results have been obtained for the control of underactuated biped with point contact [4] and [6], our strategy for walking is to control four variables, such that they track the reference defined with respect to the monotonic variable $\theta_{3}$. The four variables that are controlled are grouped in vector $h=\left[h_{1}, h_{2}, h_{3}, h_{4}\right]^{\prime}=\left[\theta_{2}-\theta_{1}, \theta_{3}-\theta_{1}+\pi, l_{1}, l_{2}\right]^{\prime}$, composed of the angle between two legs, the absolute angle of the torso, and the leg lengths, (shown in Fig.2). This vector $h$, plus $\theta_{3}$ defines the configuration of the biped. The relation with vector $\theta$ is the following:

$$
\begin{gathered}
\theta=\left[\begin{array}{c}
h_{3} \\
h_{4} \\
-h_{2}+\theta_{3} \\
h_{1}-h_{2}+\theta_{3} \\
\theta_{3}
\end{array}\right]=\left[\begin{array}{cccc}
0 & 0 & 1 & 0 \\
0 & 0 & 0 & 1 \\
0 & -1 & 0 & 0 \\
1 & -1 & 0 & 0 \\
0 & 0 & 0 & 0
\end{array}\right] h+\left[\begin{array}{l}
0 \\
0 \\
1 \\
1 \\
1
\end{array}\right]_{(10)} \\
\theta=\frac{\partial \theta}{\partial h} h+\frac{\partial \theta}{\partial \theta_{3}} \theta_{3} .
\end{gathered}
$$

where $\frac{\partial \theta}{\partial h}$ and $\frac{\partial \theta}{\partial \theta_{3}}$ are the constant matrices given in (10). Thus we have also:

$$
\ddot{\theta}=\frac{\partial \theta}{\partial h} \ddot{h}+\frac{\partial \theta}{\partial \theta_{3}} \ddot{\theta}_{3} .
$$

The control law is based on a computed torque control law and is such that the behavior of the controlled variables are:

$$
\ddot{h}=\ddot{h}^{d}-K_{p}\left(h-h^{d}\right)-K_{d}\left(\dot{h}-\dot{h}^{d}\right) .
$$

But the reference to follow is a function of the variable $\theta_{3}$ thus the reference is:

$$
\begin{aligned}
h^{d} & =h^{d}\left(\theta_{3}\right) \\
\dot{h}^{d} & =\frac{d h^{d}}{d \theta_{3}}\left(\theta_{3}\right) \dot{\theta}_{3} \\
\ddot{h}^{d} & =\frac{d h^{d}}{d \theta_{3}}\left(\theta_{3}\right) \ddot{\theta}_{3}+\frac{d^{2} h^{d}}{d \theta_{3}^{2}}\left(\theta_{3}\right) \dot{\theta}_{3}^{2},
\end{aligned}
$$

Thus the desired behavior in closed loop is given by:

$$
\begin{aligned}
\ddot{h}= & \frac{d h^{d}}{d \theta_{3}}\left(\theta_{3}\right) \ddot{\theta}_{3}+\frac{d^{2} h^{d}}{d \theta_{3}^{2}}\left(\theta_{3}\right) \dot{\theta}_{3}^{2}-K_{p}\left(h-h^{d}\left(\theta_{3}\right)\right) \\
& -K_{d}\left(\dot{h}-\frac{d h^{d}}{d \theta_{3}}\left(\theta_{3}\right) \dot{\theta}_{3}\right) .
\end{aligned}
$$

This expression is denoted:

$$
\ddot{h}=\frac{d h^{d}}{d \theta_{3}}\left(\theta_{3}\right) \ddot{\theta}_{3}+v(\theta, \dot{\theta}) .
$$

The dynamic model (1) can be expressed as function of $\ddot{h}$ and $\ddot{\theta}_{3}$ using (12)

$$
D(\theta)\left(\frac{\partial \theta}{\partial h} \ddot{h}+\frac{\partial \theta}{\partial \theta_{3}} \ddot{\theta}_{3}\right)+H(\theta, \dot{\theta})=B \Gamma,
$$

The torques will be calculated in order to have in closed loop the behavior given in (18), thus the torques must satisfy:

$$
\begin{array}{r}
D(\theta)\left(\left(\frac{\partial \theta}{\partial h} \frac{d h^{d}}{d \theta_{3}}\left(\theta_{3}\right)+\frac{\partial \theta}{\partial \theta_{3}}\right) \ddot{\theta}_{3}+\frac{\partial \theta}{\partial h} v(\theta, \dot{\theta})\right) \\
+H(\theta, \dot{\theta})=B \Gamma,
\end{array}
$$

Since the biped is underactuated, all the motion are not possible and based on the expression of matrix $B$, the admissible acceleration $\ddot{\theta}_{3}$ can be deduced. The dynamic model is decomposed into two sub-models. The first sub-model is composed of the first four lines and allows to calculate the torque. The second sub-model is composed of the fifth line and allows to calculate $\ddot{\theta}_{3}$. This sub-system gives:

$$
\ddot{\theta}_{3}=\frac{-D_{5}(\theta) \frac{\partial \theta}{\partial h} v(\theta, \dot{\theta})-H_{5}(\theta, \dot{\theta})}{D_{5}(\theta)\left(\frac{\partial \theta}{\partial h} \frac{d h^{d}}{d \theta_{3}}\left(\theta_{3}\right)+\frac{\partial \theta}{\partial \theta_{3}}\right)},
$$

where the index 5 refers to the $5^{t h}$ line of matrix $D$ and vector $H$.

Finally, the control law is obtained:

$$
\begin{aligned}
\Gamma=D_{1,4}(\theta)\left(\left(\frac{\partial \theta}{\partial h} \frac{d h^{d}}{d \theta_{3}}\left(\theta_{3}\right)+\frac{\partial \theta}{\partial \theta_{3}}\right) \ddot{\theta}_{3}\right. & \left.+\frac{\partial \theta}{\partial h} v(\theta, \dot{\theta})\right) \\
& +H_{1,4}(\theta, \dot{\theta}),
\end{aligned}
$$

where the indexes 1,4 refer to the first four lines of matrix $D$ and vector $H$.

\section{STABILITY ANALYSiS}

With the control, the output vector $h$ converges to the reference path $h^{d}\left(\theta_{3}\right)$, and if the reference function is such that the impact condition is satisfied, the output is zero step after step for convenient choice of the control gains $K_{p}, K_{d}$ [17].

\section{A. Reference path}

Since the initial and final configurations for a single support are double support configurations, when $h^{d}$ is given, $\theta_{3}$ can be deduced from geometrical relations. Thus the initial and final values of $\theta_{3}$ on one step are known and denoted $\theta_{3 i}$ and $\theta_{3 f}$. Since the condition of the impact is a geometrical condition, if the control law has converged and if $\theta_{3}$ has a monotonic evolution, the configuration at the impact is the desired one. The reference function is designed such that the impact condition is satisfied. According to equations (8), (9), and (11), the reference path must be such that:

$$
\begin{gathered}
\left(\frac{\partial \theta}{\partial h} \frac{\partial h^{d}}{\partial \theta_{3}}\left(\theta_{3 i}\right)+\frac{\partial \theta}{\partial \theta_{3}}\right) \dot{\theta}_{3 i}= \\
T_{L S} \Delta\left(\theta_{3 f}\right)\left(\frac{\partial \theta}{\partial h} \frac{\partial h^{d}}{\partial \theta_{3}}\left(\theta_{3 f}\right)+\frac{\partial \theta}{\partial \theta_{3}}\right) \dot{\theta}_{3 f} .
\end{gathered}
$$

Equality (23) is composed of five scalar equations, thus $\frac{\partial h^{d}}{\partial \theta_{3}}\left(\theta_{3 i}\right)$ and $\frac{\dot{\theta}_{3 i}}{\dot{\theta}_{3 f}}$ can be calculated as function of $\frac{\partial h^{d}}{\partial \theta_{3}}\left(\theta_{3 f}\right)$. The ration of velocities is denoted $\delta_{\dot{\theta}_{3}}$ :

$$
\delta_{\dot{\theta}_{3}}=\frac{\dot{\theta}_{3 i}}{\dot{\theta}_{3 f}} .
$$




\section{B. Principle of the stability analysis}

With the control law, the output vector $h$ converges to the reference path $h^{d}\left(\theta_{3}\right)$. In the following section we assume that $h=h^{d}\left(\theta_{3}\right)$, that is, the system tracks the reference path. The five degrees of freedom (DoF) of the biped can be reduced to one DoF of a virtual equivalent pendulum under the condition, and we will hence analyze stability of the pendulum instead of the original biped.

This condition does not mean that the biped motion is cyclic with respect to time since the temporal evolution of $\theta_{3}$ is the result of integration of equation (21), and thus depends on the reference path $h^{d}\left(\theta_{3}\right)$. For a SS phase $\theta_{3}$ must evolve monotonically from $\theta_{3 i}$ to $\theta_{3 f}$. The temporal evolution of the biped during a SS phase is completely defined by the velocity $\dot{\theta}_{3}$ for one particular value $\theta_{3}$. The stability analysis is based on the Poincaré return map, and this return map will be built just before the impact, when the biped is in the configuration $h^{d}\left(\theta_{3 f}\right), \theta_{3 f}$. The variable that is effective to study the convergence to a cyclic motion is $\dot{\theta}_{3 f}$. Since the angular momentum is proportional to $\dot{\theta}_{3 f}$, the angular momentum (or its square value) can also be used in the stability analysis

\section{SS phase}

According the Newton-Euler second law, the equilibrium of the biped in rotation around the mobile contact point $N_{1}$ gives:

$$
\dot{\sigma}_{N_{1}}+M V_{N_{1}} \times V_{G}=\overrightarrow{N_{1}} G \times M \vec{g},
$$

where $V_{N_{1}}$ and $V_{G}$ are the velocities at the points $N_{1}=$ $\left[-R \theta_{3}, 0\right]^{\prime}$ and the center of mass, $G=\left[x_{G}, y_{G}\right]^{\prime}, M$ is the total mass of the biped, the gravity vector is $\vec{g}=[0,-g]^{\prime}$, and $\sigma_{N_{1}}$ is the angular momentum about $N_{1}$ because the gravity is the only external force that produces a torque around $N_{1}$. By defining $\sigma_{N_{1}}$ is:

$$
\sigma_{N_{1}}=\sum_{i} m_{i} \overrightarrow{N_{1} G_{i}} \times V_{G i}+\sum_{i} I_{i} w_{i}
$$

where $G_{i}$ is the center of mass for the link $i, m_{i}$ and $I_{i}$ are the mass and the inertia of link $i, w_{i}$ is the angular velocity of link $i$, and $V_{G i}$ is the linear velocity of $G_{i}$. This quantity is linear with respect to the joint velocity component and can be written:

$$
\sigma_{N_{1}}=S(\theta) \dot{\theta}
$$

We assume that the biped follows reference path thus we have:

$$
\begin{aligned}
\theta & =\frac{\partial \theta}{\partial h} h^{d}\left(\theta_{3}\right)+\frac{\partial \theta}{\partial \theta_{3}} \theta_{3} . \\
\dot{\theta} & =\frac{\partial \theta}{\partial h} \frac{\partial h^{d}}{\partial \theta_{3}}\left(\theta_{3}\right) \dot{\theta}_{3}+\frac{\partial \theta}{\partial \theta_{3}} \dot{\theta}_{3} .
\end{aligned}
$$

Thus the angular momentum $\sigma_{N_{1}}$ (27) is rewritten:

$$
\sigma_{N_{1}}=S(\theta)\left(\frac{\partial \theta}{\partial h} \frac{\partial h^{d}}{\partial \theta_{3}}\left(\theta_{3}\right)+\frac{\partial \theta}{\partial \theta_{3}}\right) \dot{\theta}_{3}=I_{\theta_{3}}\left(\theta_{3}\right) \dot{\theta}_{3} .
$$

Equation (25) can be developed using the expression of $\overrightarrow{N_{1}} G, V_{G}, V_{N_{1}}$ as:

$$
\dot{\sigma}_{N_{1}}=-M g\left(x_{G}\left(\theta_{3}\right)+R \theta_{3}\right)+M R \frac{d y_{G}\left(\theta_{3}\right)}{d \theta_{3}} \dot{\theta}_{3}^{2} .
$$

Equation (30) is combined to equation (31) to express the derivative of $\sigma_{N_{1}}$ with respect to $\theta_{3}$, under the assumption that $\theta_{3}$ is monotonic:

$$
\frac{d \sigma_{N_{1}}}{d \theta_{3}}=-M g\left(x_{G}+R \theta_{3}\right) \frac{I_{\theta_{3}}}{\sigma_{N_{1}}}+M R \frac{d y_{G}}{d \theta_{3}} \frac{\sigma_{N_{1}}}{I_{\theta_{3}}} .
$$

A new variable $\xi=\sigma_{N_{1}}^{2} / 2$ is introduced, to transform equation (32) into an equation that can be integrated analytically:

$$
\begin{aligned}
\frac{d \xi}{d \theta_{3}} & =\kappa_{1}\left(\theta_{3}\right)+2 \kappa_{2}\left(\theta_{3}\right) \xi, \\
\kappa_{1}\left(\theta_{3}\right) & =-M g\left(x_{G}+R \theta_{3}\right) I_{\theta_{3}}, \\
\kappa_{2}\left(\theta_{3}\right) & =\frac{M R}{I_{\theta_{3}}}\left(\frac{\partial y_{G}(\theta)}{\partial \theta}\right)^{\prime} \frac{d \theta^{d}}{d \theta_{3}} .
\end{aligned}
$$

Equation (33) is a first order ordinary differential equation linear in $\xi$. Therefore, a general solution for the initial value $\theta_{3 i}$ can be obtained:

$$
\begin{aligned}
\xi\left(\theta_{3}\right) & =\delta_{S S}^{2}\left(\theta_{3}\right) \xi\left(\theta_{3 i}\right)+V\left(\theta_{3}\right) \\
\delta_{S S}\left(\theta_{3}\right) & =\exp \left(\int_{\theta_{3 i}}^{\theta_{3}} \kappa_{2}\left(\tau_{2}\right) d \tau_{2}\right) \\
V\left(\theta_{3}\right) & =\int_{\theta_{3 i}}^{\theta_{3}} \exp \left(\int_{\tau_{1}}^{\theta_{3}} 2 \kappa_{2}\left(\tau_{2}\right) d \tau_{2}\right) \kappa_{1}\left(\tau_{1}\right) d \tau_{1}
\end{aligned}
$$

$\xi$ and $V$ are a pseudo-kinetic and a pseudo-potential energies of the virtual equivalent pendulum, respectively.

As a consequence if $\dot{\theta}_{3 i}$ is known $\dot{\theta}_{3}$ can be deduced for the current step as a function of $V$ and $\delta_{S S}$ without integration of (25). To be able to deduce from this equation the evolution of $\xi$ (and in consequence of $\sigma_{N_{1}}$ and $\dot{\theta}_{3}$ ) step after step, the evolution of $\xi$ at the impact must be taken into account. In the following section, the index $k$ will be added to denote the number of the current step

\section{Impact phase}

Let us consider the impact between steps $k$ and $k+1$. Using (30), $\xi$ at the end of step $k$ is:

$$
\xi_{k}\left(\theta_{3 f}\right)=\frac{1}{2}\left(I_{\theta_{3 f}}\left(\theta_{3 f}\right) \dot{\theta}_{3 f, k}\right)^{2}
$$

and $\xi$ at the beginning of the step $k+1$ is:

$$
\xi_{k+1}\left(\theta_{3 i}\right)=\frac{1}{2}\left(I_{\theta_{3 i}}\left(\theta_{3 i}\right) \dot{\theta}_{3 i, k+1}\right)^{2}
$$

Using (24), and defining $\delta_{I}$ by,

$$
\delta_{I}=I_{\theta_{3}}\left(\theta_{3 i}\right) / I_{\theta_{3}}\left(\theta_{3 f}\right),
$$

we obtain:

$$
\xi_{k+1}\left(\theta_{3 i}\right)=\delta_{I}^{2} \delta_{\dot{\theta}_{3}}^{2} \xi_{k}\left(\theta_{3 f}\right) .
$$




\section{E. Poincaré map}

Combining (34) and (40), the final value of $\xi$ from the $k$ th step to the $k+1$ th step is as follows:

$$
\begin{aligned}
\xi_{k+1}\left(\theta_{3 f}\right) & =\delta^{2}\left(\theta_{3 f}\right) \xi_{k}\left(\theta_{3 f}\right)+V\left(\theta_{3 f}\right) \\
\delta\left(\theta_{3 f}\right) & =\delta_{S S}\left(\theta_{3 f}\right) \delta_{I} \delta_{\dot{\theta}_{3}}
\end{aligned}
$$

where $\theta_{3 f}$ is the value of $\theta_{3}$ just before the impact. This equation describes the Poincaré map that we choose.

If a cyclic motion exists, then $\xi_{k+1}\left(\theta_{3 f}\right)$ corresponds to $\xi_{k}\left(\theta_{3 f}\right)$. Thus, a fixed point $\xi_{c}\left(\theta_{3 f}\right)$ is given using (41) as follows:

$$
\xi_{c}\left(\theta_{3 f}\right)=\frac{V\left(\theta_{3 f}\right)}{1-\delta^{2}\left(\theta_{3 f}\right)} .
$$

Since $\xi_{c}\left(\theta_{3 f}\right)$ is positive, $V\left(\theta_{3 f}\right)$ and $1-\delta^{2}\left(\theta_{3 f}\right)$ must have the same sign. The following cases can occur:

Case 1: From (41), the fixed point is stable, if $\delta^{2}\left(\theta_{3 f}\right)<1$. Therefore, if $\delta^{2}\left(\theta_{3 f}\right)<1$ and $V\left(\theta_{3 f}\right)>0$, then an asymptotically stable cyclic motion exists.

Case 2: If $\delta^{2}\left(\theta_{3 f}\right)=1$ and $V\left(\theta_{3 f}\right)=0$, from (41), $\xi_{k+1}\left(\theta_{3 f}\right)=\xi_{k}\left(\theta_{3 f}\right)$, namely, all motions are cyclic.

Case 3: From (41), the fixed point is unstable, if $\delta^{2}\left(\theta_{3 f}\right)>1$. Therefore, if $\delta^{2}\left(\theta_{3 f}\right)>1$ and $V\left(\theta_{3 f}\right)<0$, then an unstable cyclic motion exists.

Since by definition $\xi \geq 0$, from equation (41) for the complete step, $\xi_{c}$ must satisfy the following inequality:

$$
\xi_{c}\left(\theta_{3 f}\right) \geq \max _{\theta_{3}} \frac{-V\left(\theta_{3}\right)}{\delta^{2}\left(\theta_{3}\right)} .
$$

to have a monotonic evolution of $\theta_{3}$.

Since a product of the two variables $\left(\delta_{I} \cdot \delta_{\dot{\theta}_{3}}\right)$ is the ratio of momentum $\sigma_{N_{1}}$ at the contact point $N_{1}$ before and after the impact, the speed of convergence is mainly associated with this ratio (we will see in the following sections), and connected to the distance between the contact points and velocity of the mass center before the impact [19].

The contact point before the impact, at the end of the single support phase, is denoted $N_{1}$, the contact point after the impact, at the beginning of the next single support phase, is denoted $N_{2}$. Therefore there is a change of contact point between the two single support phases, where the angular momentum is calculated. At the impact, both legs stay on the ground with the contact points $N_{1}$ and $N_{2}$. Then is it possible to compute equilibrium relation of the biped at the impact to study the cyclic behavior in function of the value of radii. Let us detail this question.

The distance $d$ between the $N_{1}$ and $N_{2}$ is (see Fig.3)

$$
N_{1} N_{2}=d=2(l-R) \sin \left(h_{1} / 2\right) .
$$

The angular momentum before the impact denoted $\sigma_{N_{1}}^{-}$is calculated around $N_{1}$ and can also be calculated around $N_{2}$, it is then denoted $\sigma_{N_{2}}^{-}$, the angular momentum transfer gives:

$$
\sigma_{N_{2}}^{-}=\sigma_{N_{1}}^{-}-M \cdot d \cdot \dot{y}_{G}^{-}
$$

TABLE I

PHYSICAL PARAMETERS FOR THE DYNAMIC MODEL

\begin{tabular}{cc|cc|cc|cc}
\hline$m_{s}$ & $1[\mathrm{~kg}]$ & $I_{s}$ & $0.05_{\left[\mathrm{kgm}^{2}\right]}$ & $s_{h}$ & $0.4[\mathrm{~m}]$ & $l_{1}$ & $0.8 \sim 0.85[\mathrm{~m}]$ \\
$m_{f}$ & $1[\mathrm{~kg}]$ & $I_{f}$ & $0.05_{\left[\mathrm{kgm}^{2}\right]}$ & $f_{m}$ & $0.2[\mathrm{~m}]$ & $l_{2}$ & $0.75 \sim 0.8[\mathrm{~m}]$ \\
$m_{b}$ & $15[\mathrm{~kg}]$ & $I_{b}$ & $3_{\left[\mathrm{kgm}^{2}\right]}$ & $s_{b}$ & $0.1[\mathrm{~m}]$ & $R$ & $0 \sim 1.0[\mathrm{~m}]$ \\
\hline
\end{tabular}

At the impact, considering the vertical component $I_{m y_{1}}$ of the impulsive ground reaction $I_{m_{1}}$ in the point $N_{1}$, the equilibrium in rotation around $N_{2}$ gives:

$$
\sigma_{N_{2}}^{+}=\sigma_{N_{2}}^{-}-d \cdot I_{m y_{1}}
$$

Considering the vertical components $I_{m y_{1}}$ and $I_{m y_{2}}$ of the impulsive ground reactions $I_{m_{1}}$ and $I_{m_{2}}$ respectively in the points $N_{1}$ and $N_{2}$, the vertical equilibrium of the biped at the impact is :

$$
I_{m y_{1}}+I_{m y_{2}}=M\left(\dot{y}_{G}^{+}-\dot{y}_{G}^{-}\right),
$$

The impact are such that the two legs stay on the ground, thus $I_{m y_{1}}>0$ and $I_{m y_{2}}>0$ and we have:

$$
0<I_{m y_{1}}<M\left(\dot{y}_{G}^{+}-\dot{y}_{G}^{-}\right) .
$$

As a consequence, combining (46), (47), and (49), we have:

$$
\begin{array}{rr}
\sigma_{N_{1}}^{-}-M \cdot d \cdot \dot{y}_{G}^{+}<\sigma_{N_{2}}^{+}<\sigma_{N_{1}}^{-}-M \cdot d \cdot \dot{y}_{G}^{-}, & \text {if } d>0, \\
\sigma_{N_{2}}^{+}=\sigma_{N_{1}}^{-}, & \text {if } d=0, \\
\sigma_{N_{1}}^{-}-M \cdot d \cdot \dot{y}_{G}^{-}<\sigma_{N_{2}}^{+}<\sigma_{N_{1}}^{-}-M \cdot d \cdot \dot{y}_{G}^{+}, & \text {if } d<0 .
\end{array}
$$

Since $I_{\theta_{3}}>0$ (see Fig.8) and $\dot{\theta}_{3}<0$ (see Fig.5), $\sigma_{N_{1}}^{-}<0$. Considering (24), (30) and (39), the ratio $\delta_{I} \delta_{\dot{\theta}_{3}}$ is bounded:

$$
\begin{array}{r}
1-M \cdot d \cdot \frac{\dot{y}_{G}^{-}}{\sigma_{N_{1}}^{-}}<\delta_{I} \delta_{\dot{\theta}_{3}}<1-M \cdot d \cdot \frac{\dot{y}_{G}^{+}}{\sigma_{N_{1}}^{-}}, \quad(d>0), \quad(d=0), \\
\delta_{I} \delta_{\dot{\theta}_{3}}=1, \quad(d<0) \\
1-M \cdot d \cdot \frac{\dot{y}_{G}^{+}}{\sigma_{N_{1}}^{-}}<\delta_{I} \delta_{\dot{\theta}_{3}}<1-M \cdot d \cdot \frac{\dot{y}_{G}^{-}}{\sigma_{N_{1}}^{-}}, \quad(d<
\end{array}
$$

\section{Simulation}

In simulations, the physical parameters of the biped shown in Fig. 2 are used (see Table I). The gains of the control law are chosen so that tracking errors can be smaller than $10^{-4}$ for all walking gaits (shown in Eq.(56)).

$$
\left\{\begin{array}{l}
K_{p}=\operatorname{diag}\left(\left[10^{5}, 10^{4}, 10^{5}, 5 \times 10^{4}\right]\right) \\
K_{d}=\operatorname{diag}\left(\left[5 \times 10^{2}, 5 \times 10^{2}, 10^{3}, 5 \times 10^{2}\right]\right)
\end{array}\right.
$$

Fig.4 shows examples of stick diagrams of walking for one step with the foot radii $R=0[\mathrm{~m}], 0.2[\mathrm{~m}], 0.5[\mathrm{~m}]$ and $0.7[\mathrm{~m}]$ and the step angle $=0.24$ [rad]. A cyclic motion for $R=0.5$ [m] is given in Fig.5. CoM positions with respect to $R$ are shown in Fig.6. Tangent vectors of right ends of lines are expressing a post-impact velocity of CoM. The variation of CoM velocities at the impact are presented in Fig.7. 

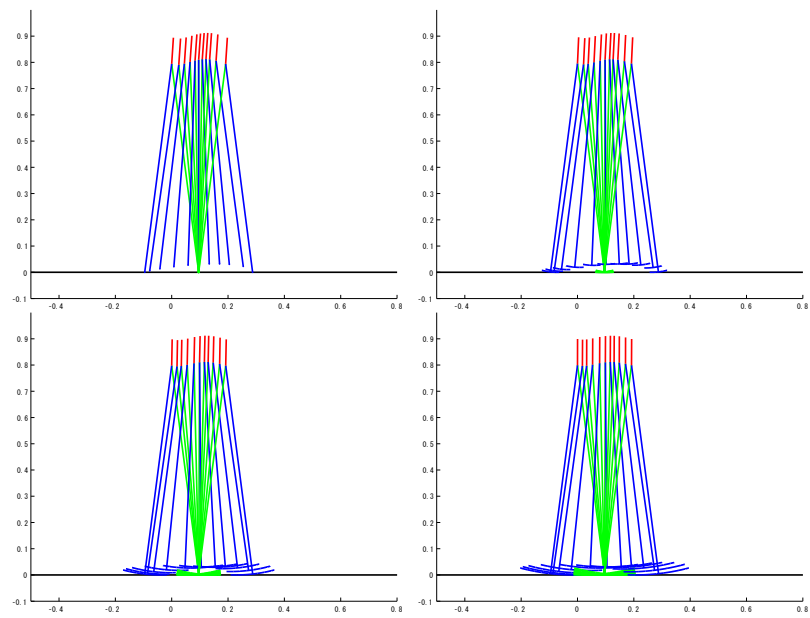

Fig. 4. The stick diagrams of walking. The foot radii $R=0[\mathrm{~m}], 0.2[\mathrm{~m}]$, $0.5[\mathrm{~m}]$ and $0.7[\mathrm{~m}]$.
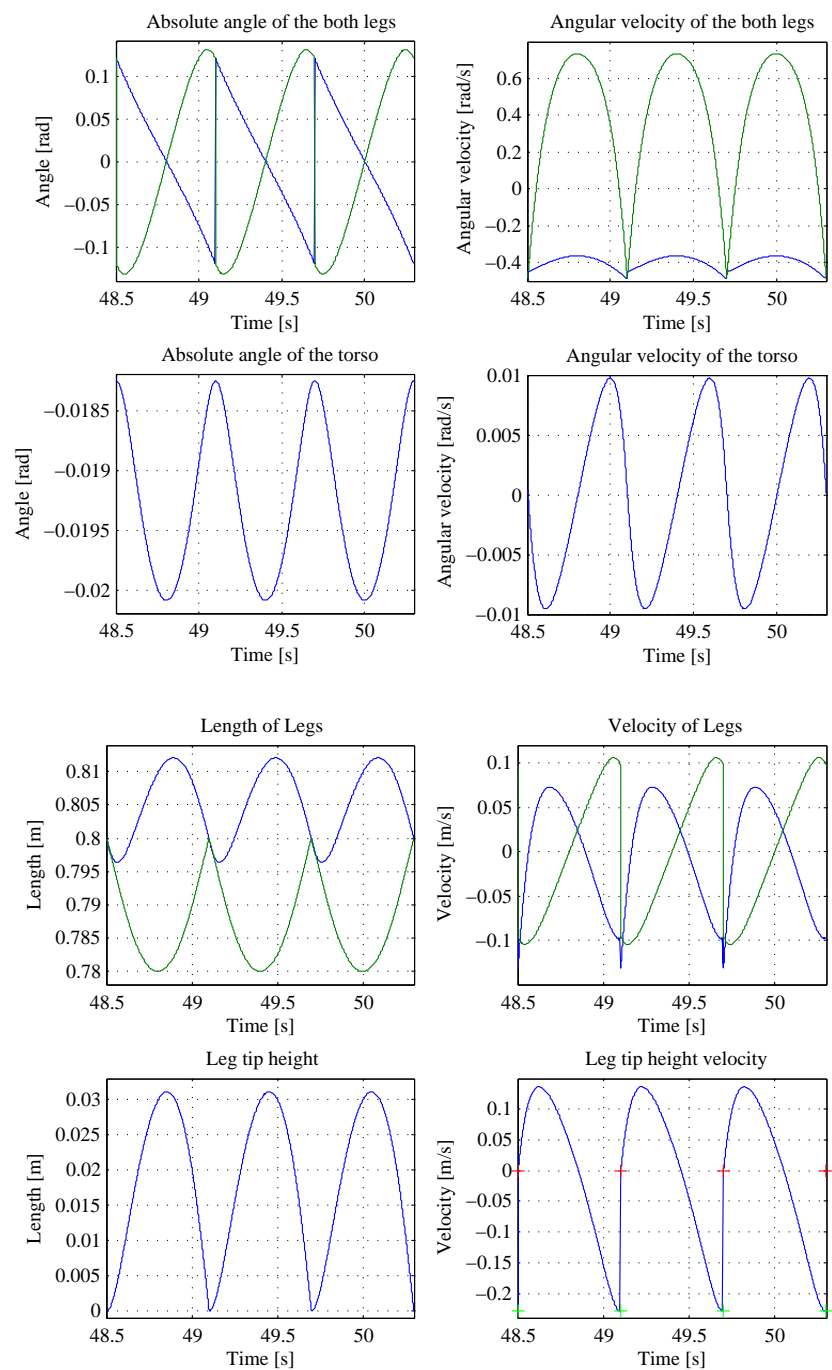

Fig. 5. Time responses at the cyclic motion with $R=0.5[\mathrm{~m}]$ of the angle of the both legs, the torso, the length of legs and the leg tip. The reference paths are almost identical to the time responses.
TABLE II

TORSO ANGLES. THE ANGLES ARE CHOSEN SUCH THAT CYCLIC MOTIONS HAVE THE SAME VALUE $\xi_{c}\left(\theta_{3 f}\right)=\xi(-0.12)=16.27$.

\begin{tabular}{c|ccccc}
\hline Foot radius [m] & 0 & 0.1 & 0.2 & 0.3 & \\
Angle of torso [rad] & -0.060 & -0.051 & -0.043 & -0.034 & \\
\hline Foot radius [m] & 0.4 & 0.5 & 0.6 & 0.7 & 0.8 \\
Angle of torso [rad] & -0.026 & -0.018 & -0.011 & -0.004 & 0.002 \\
\hline
\end{tabular}

\section{A. Design of Reference Path}

The reference path $h^{d}$ is defined by a fourth order polynomial function such that:

$$
h_{d}\left(\theta_{3}\right)=a\left[1, \theta_{3}^{1}, \theta_{3}^{2}, \theta_{3}^{3}, \theta_{3}^{4}\right]^{\prime},
$$

where $a \in \Re^{4 \times 5}$ is a coefficient matrix for the reference $h_{d}$. An intermediate position of SS phase, positions and velocities just before and after the impact are given in order to calculate the coefficients of the reference paths (see Fig.4).

Walking is depending on not only the radii of feet but also of the reference path of the length of the legs. The foot radius reduces the velocity of the CoM before the impact. The reference paths of the legs are chosen to smoothen the vertical variation of the CoM. However the references of the legs are affected by the impact, and the choice of the reference paths is limited accordingly. The radius mainly smoothens the vertical CoM motion.

The initial and the final length for the legs are chosen as the same value. The final velocity for the biped are arbitrary fixed. The intermediate configuration for the legs is chosen such that the swing leg length decreases $0.02 \mathrm{~m}$ and the stance leg length increases $0.01 \mathrm{~m}$ during the step to avoid that the swing leg tip touches the ground and the length of the leg is $0.8[\mathrm{~m}]$ at the impact. Therefore the top position of the CoM is almost same for each foot radius as shown in Fig.6. For one value $R$, we choose the angle of the torso at the impact arbitrary. The angle of the torso at the intermediate configuration is equal to $110 \%$ of the value of the torso angle at the impact. The states of the biped to begin the step, $\theta_{i}, \frac{\partial h^{d}}{\partial \theta_{3}}$, and $\delta_{\dot{\theta}_{3}}$ are deduced using the impact equation (8) and the corresponding value $\xi_{c}\left(\theta_{3 f}\right)$ is deduced.

Then from this reference motion we deduced the reference motion for the other value of the radius $R$. For example, the coefficient matrix in Eq.(57) for $R=0.5$ is obtained as follows:

$$
\begin{aligned}
& \left.a\right|_{R=0.5}= \\
& {\left[\begin{array}{ccccc}
0 & -3.02 & -0.158 & 70.8 & 10.9 \\
-0.0201 & 0.0002 & 0.255 & -0.0106 & -8.89 \\
0.810 & -0.122 & -1.58 & 8.50 & 61.2 \\
0.780 & -0.0037 & 1.91 & 0.254 & -36.5
\end{array}\right]}
\end{aligned}
$$

The angle of the torso at the impact $h_{2}\left(\theta_{3 f}\right)$ is adjusted such that the cyclic motions for all foot radii $R$ have the same value $\xi_{c}\left(\theta_{3 f}\right)$ as shown in Table II. Energy excitation for continuous walking with smaller feet radius is mainly done 

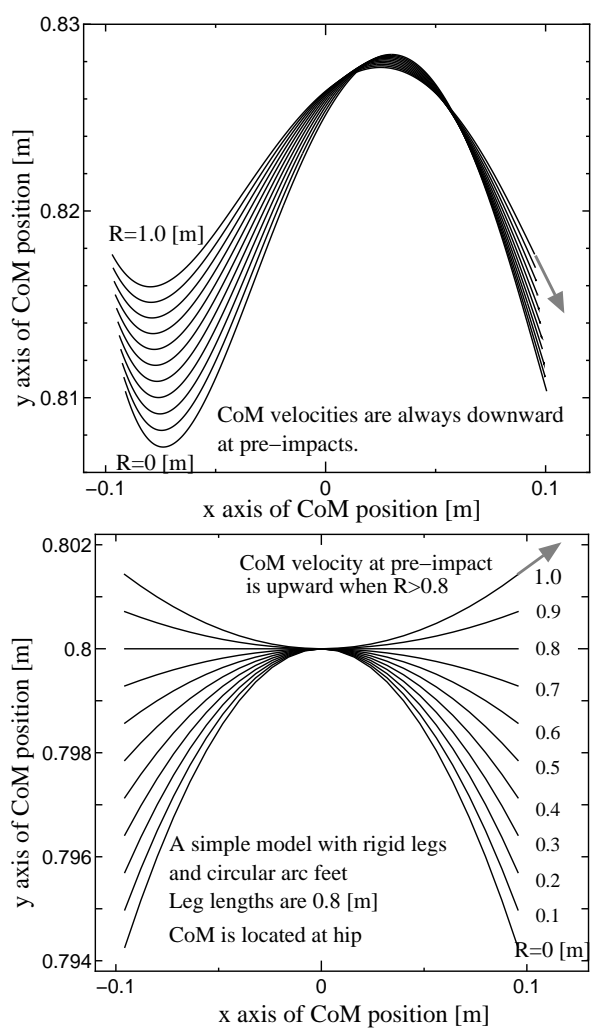

Fig. 6. CoM positions with respect to R. Upper: the case of our biped shown in Fig.2. Tangent vectors of right ends of lines are expressing a post-impact velocity of CoM. Lower: the case of a simple model with rigid legs and circular arc feet. CoM is located at hip position. When $\mathrm{R}>0.8$ [m], CoM velocities are upward. It gives a contradiction at the impact or there would be a flight phase.

by the asymmetric mass distribution due to the torso forward inclination. Leg swing also provides a way of putting energy. For small feet radii, the energy for walking is produced by the weight of the torso that is inclined forward. For larger feet radii, the energy for walking is produced by the motion of the swing leg.

Since the impact equation changes, the initial configuration and velocity are changed accordingly. During the impact, for the chosen reference path, the two legs stay on the ground.

\section{B. Stability Analysis}

The variables in the analytic solution (34) are shown in Fig. 8 with respect to the monotonic variable $\theta_{3}$ for variation of the foot radius $R$. It should be noted that the monotonic variable is evolving from a positive value to a negative value, $\theta_{3}: 0.12$ [rad] $\rightarrow-0.12$ [rad]. In the lower right part of Fig.8, $\xi_{c}(\theta)$ is given for all the cyclic motions. It can be observed that $\xi_{c}\left(\theta_{3 f}\right)=\xi(-0.12)=16.27$. The upper left part of the figure presents $\delta_{S S}^{2}\left(\theta_{3}\right)$ is given by equation (35). The convergence of Poincaré map, as shown in equation (42), is function of $\delta_{S S}^{2}\left(\theta_{3 f}\right)=\delta_{S S}^{2}(-0.12)$. However the values of $\delta_{S S}^{2}(-0.12)$ are very close to unit thus the convergence of Poincare map is essentially defined by the impact map : $\delta\left(\theta_{3 f}\right) \approx \delta_{I} \delta_{\dot{\theta}_{3}}$. The upper right part of Fig. 8 represents the evolution of $V$ defined

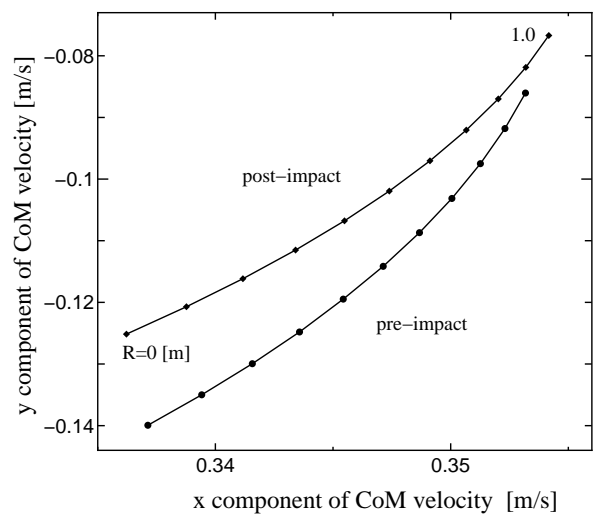

Fig. 7. CoM velocities at the impact with respect to $R$. The point corresponds to different value of $R$ from 0 to 1 , the abscissa of the point gives the horizontal velocity $\dot{x}_{G}$ respectively before and after the impact, the ordinates gives the vertical velocity $\dot{y}_{G}$ respectively before and after the impact. The vertical velocities before the impact are always directed downward.

by equation (36). These functions are essentially affected by the evolution $\xi$. The lower left part of Fig.8 shows the term $I_{\theta_{3}}$ given by equation (30), $I_{\theta_{3}}$ is always positive and has not large variation.

This first study concerns reference path with an interlink angle at the impact equals to 0.24 [rad]. For this value, the evolution of $\delta_{S S}^{2}\left(\theta_{3 f}\right), \delta_{I}, \delta_{\dot{\theta}_{3}}$ and $\delta\left(\theta_{3 f}\right)$ are given in solid line in Fig.9, as function of the $R$. The cyclic motion is stable for $R<0.8$.

In order to determine if the radius $R=0.8$ is a limit of stability only for one specific reference path or if this limit is more physical, different kinds of reference motion are considered in the following. Only the interlink angle $h_{1}\left(\theta_{3 f}\right)$ at the impact is changed. For different values of $h_{1}$ and radii $R$, the coefficient involves in the convergence condition are drawn in Fig.9.

$\delta_{\dot{\theta}_{3}}$ in upper right part of the figure and $\delta_{I}$ in lower left part of the figure increase when $R$ increases and $h_{1}\left(\theta_{3 f}\right)$ decreases. $\delta^{2}$ also increases at the same time (lower right in Fig.9). The term $\delta^{2}$ comes to unit when $R=0.8[\mathrm{~m}]$ which means that $R$ has the same values as the length of legs at the impact.

Remark: We confirmed in another simulations that variations of the torso angle had small influences on $\delta_{I}$ and $\delta_{\dot{\theta}_{3}}$ although it essentially affects $\xi$. The variables $V, \delta_{S S}, I_{\theta_{3}}$ and $\xi$ in the analytic solution for SS phase change for the torso angle. However the variation of $\delta_{S S}$ is smaller than the variations of $\delta_{I}$ and $\delta_{\dot{\theta}_{3}}$ with respect to the foot radii.

Fig.10 presents the stability property with respect to the foot radii. Two black rigid lines show $V$ and $\delta^{2}-1 . V$ and $\delta^{2}-1$ have opposite sign thus a cyclic motion may exist such that (44) is satisfied for any value of radii $R$. For $R<0.8[\mathrm{~m}]$, the motion is stable. For $R>0.8[\mathrm{~m}]$, the motion is unstable. For $R=0.8[\mathrm{~m}]$, the motion is neutral, in this case any value $\xi_{c}$ produces cyclic motions.

Case corresponding to a radius superior to the length of each leg, $(R>0.8[\mathrm{~m}])$ can be studied if we consider the motions of feet are not in the same sagittal plane to avoid collisions. In 

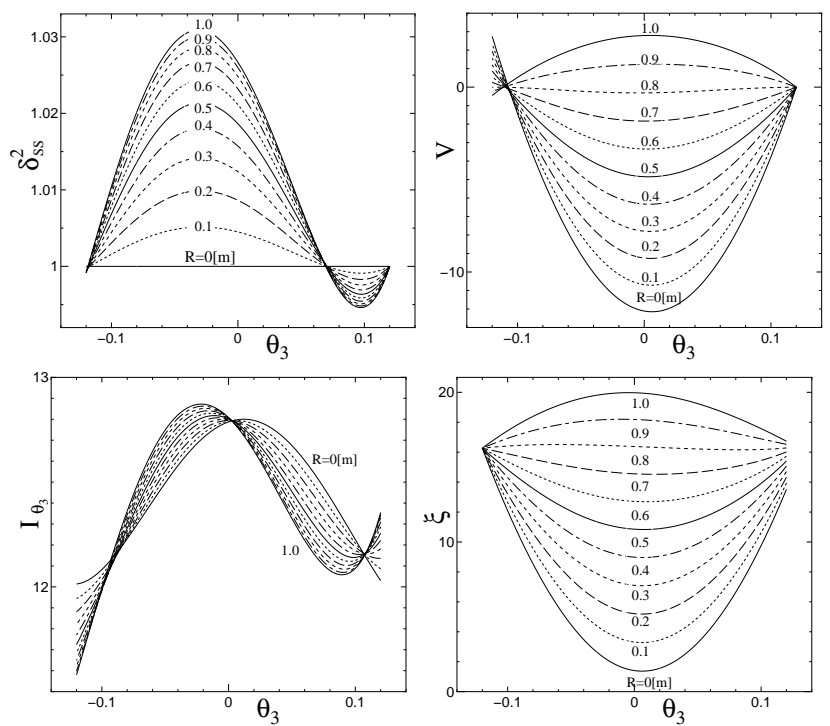

Fig. 8. Analytic solutions for SS phase. The figures are $\delta_{S S}^{2}$ by Eq.35, $V$ by Eq.36, the function $I_{\theta_{3}}$ by Eq.30 and $\xi$ by Eq.34. $\theta_{3}$ evolves from positive $(0.12)$ to negative $(-0.12)$

the leg exchange, at the impact, the contact point moves back but the contact point has a large forward progression during the single support phase, the biped goes forward.

The gradient $\delta^{2}$ (Eq. 42) of Poincaré map (Eq. 41) depends on the SS phase $\left(\delta_{S S}\right)$ and the impact phase $\left(\delta_{I} \cdot \delta_{\dot{\theta}_{3}}\right) . \delta_{S S}$ was close to unit at the impact. Since $\dot{y}_{G}^{-}<\dot{y}_{G}^{+}<0$ (see Fig.7), we obtain that the foot radius $R$ and the sign of $d$ defined the position of the ratio $\delta_{I} \delta_{\dot{\theta}_{3}}$ with respect to 1 from Eq.(53) to Eq.( 55).

- if $R<l, d>0$, and $\delta_{I} \delta_{\dot{\theta}_{3}}<1$

- if $R=l, d=0$, and $\delta_{I} \delta_{\dot{\theta}_{3}}=1$

- if $R>l, d<0$, and $\delta_{I} \delta_{\dot{\theta}_{3}}>1$

The property of the gradient $\delta^{2}$ agrees with "speed mode" of passive dynamic walking obtained by McGeer [11]. Wisse [14] finds results that are different from our results. For passive walking he finds that for stability point of view the best radius is $14 \%$ of leg length, this value corresponds to a case where two monotonic lines of eigenvalues are crossing. The increasing one is represented 'Speed mode', and the decreasing one is 'Totter mode'. However the crossing point changes with respect to slope angle and physical parameters of bipeds. The $14 \%$ of leg length is not the best radius, generally speaking. In our controlled system, it is predictable that the 'Totter mode' is close to zero or much smaller than the 'Speed mode', since the 'Speed mode' is expressed by the zero dynamics of the controlled system and the 'Totter mode' is depending on the controller gains. Term $\delta^{2}$ has the same property of the 'Speed mode', and thus is increasing with respect to $R$.

\section{Basin of Attraction}

Basins of attraction determined by numerical computations are shown in Fig.11. The larger the foot radii are in the stable domain, the wider the basin of attraction is but the slower the
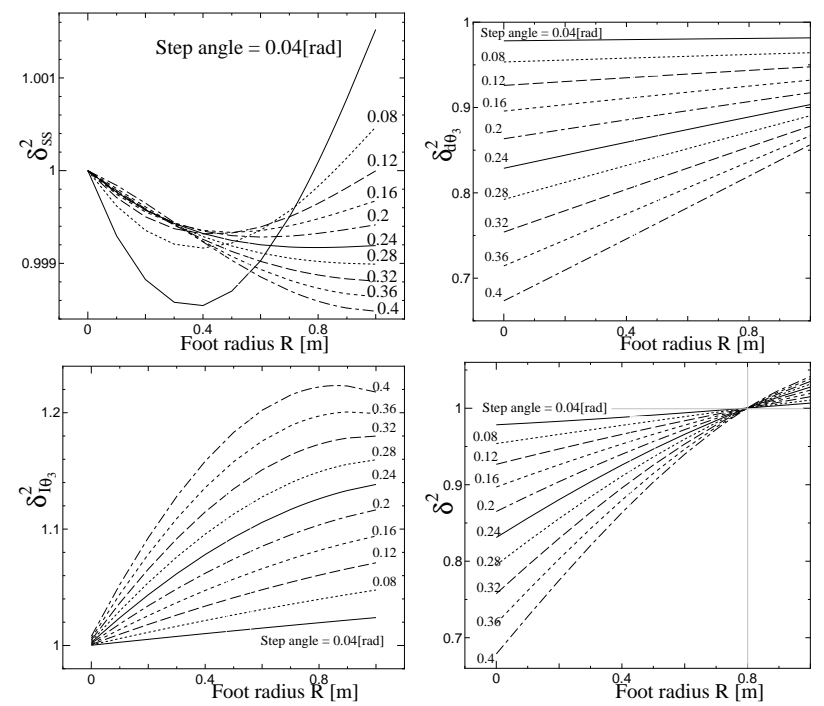

Fig. 9. Slope of the Poincaré return map. Step angle which means angles between two legs at the impact varies from $0.04[\mathrm{rad}]$ to 0.40 [rad]. The figures show $\delta_{S S}^{2}$ (upper left), $\delta_{\dot{\theta}_{3}}^{2}$ (upper right), $\delta_{I_{\theta_{3}}}^{2}$ (lower left) and $\delta^{2}$ (lower right) with respect to the foot radii $R=0 \stackrel{\sim}{\sim} 1.0[\mathrm{~m}] . R=0.8$ $[\mathrm{m}]$ means that the radius is the same as the leg length at the impact for the analytic solution. For $R=0.8[\mathrm{~m}]$, the cyclic motion is not stable.

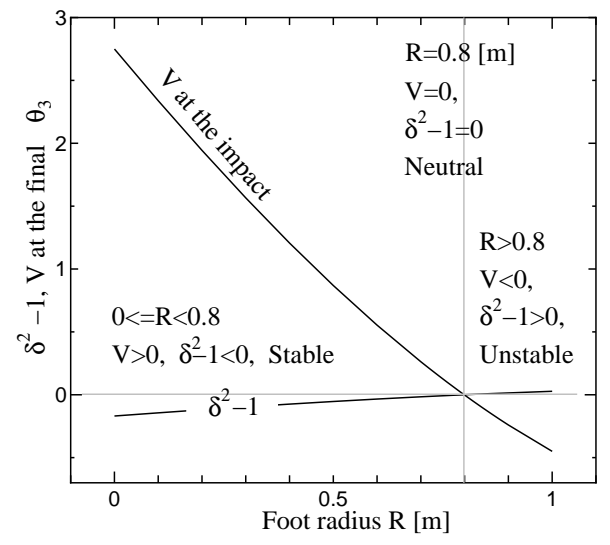

Fig. 10. The property of stability with respect to the foot radii $R$. Two black rigid lines show $V$ and $\delta^{2}-1 . V$ and $\delta^{2}-1$ have opposite sign thus a cyclic motion may exist such that (44) is satisfied. For $R<0.8[\mathrm{~m}]$ the motion is stable. For $R>0.8[\mathrm{~m}]$ the motion is unstable. For $R=0.8[\mathrm{~m}]$ the motion is neutral, that is all of $\xi_{c}$ are cyclic motions.

speed of convergence is. If the foot radius is the same as the leg length, the motion is neutral, that is, all motions are cyclic.

In Fig.11, the area between the line of $\xi_{\min }^{-}$and $\xi_{\max }^{-}$is the basin of attraction. The variable $\xi$ just before the impact is used for expressing the basin of attraction. The line $\xi_{c}$ represents the cyclic motions. Fig.12 presents time evolutions of $\theta_{3}, \dot{\theta}_{3}$ for 100 steps. For top to bottom, the following foot radii are considered $R=0[\mathrm{~m}], 0.5[\mathrm{~m}], 0.8[\mathrm{~m}]$ and $1.3[\mathrm{~m}]$ from the top figure. The first two cases are clearly stable, the third one is neutral, and the last one is unstable. Simulations confirm the existence of the neutral condition.

The property of the basin of attraction with respect to the radius is also analogous to the results of passive dynamic 
walking by Wisse [12]. As depicted in Fig.11, the bottom line shows the theoretic line of a minimal $\xi$ corresponding to the numerical one. The theoretic results comes from Eq.(44). It means a required minimal angular momentum to overcome a gap from a minimum of a vertical position of CoM to a maximum. If the momentum is smaller than the minimum, the complete step is not achieved, the step begins and then the robot goes backward to return to its initial configuration for the step. After that, the robot stops, but it does not fall down contrarily to a passive dynamic walker [13] that falls down backward.

From Fig.6, the smaller the radius is, the larger the gaps of the vertical positions of $\mathrm{CoM}$ and the minimal $\xi_{\min }^{-}$are. Thus the circular arc feet broaden the minimal bounds. The variation of the maximal bounds is caused by properties of vertical reaction forces. The reaction force vector $R_{1}$ at the point $N_{1}$ is given by the following equation:

$$
R_{1}=\left[\begin{array}{l}
R_{x_{1}} \\
R_{y_{1}}
\end{array}\right]=\left[\begin{array}{l}
M \ddot{x}_{G} \\
M\left(\ddot{y}_{G}+g\right)
\end{array}\right] .
$$

The vertical acceleration $\ddot{y}_{G}$ is decided by the the centrifugal force caused by the angular velocity of the stance leg $\dot{\theta}_{3}$ and an acceleration of the leg variation $\ddot{l}_{i}(t)$. The radius smoothens the variation of CoM, and consequently the centrifugal force is reduced. We observe that the acceleration of the leg is smaller when the radii increase. Thus, the maximal $\xi_{\max }^{-}$is extended when the radius increases. Our controller ensures stability from the minimal boundary line to the maximum for all radii. Namely, the basin of attraction is broaden by physical properties such as the feet radii. Globally, our controlled system has similar properties for stability and basin of attraction to the passive dynamic walking.

A large foot radius can reduce the variation of the CoM during steps. We can summarize that the basin of attraction is broadened by a reduction of the CoM variation for the larger radius. The difference might be vanished by the leg length variation. To satisfy the velocity jump condition through the impact, some amounts of a CoM variation are needed for the reference path. In the simulation, the variation shown in Fig.6 gave one of the smallest CoM variation.

\section{Consumed Energy}

Consumed energies for one cyclic step with respect to the foot radii $R$ is described in Fig.13. The following formula is used for computing the consumed energy:

$$
E_{c}=\int_{0}^{T}\left|\dot{\theta}^{\prime} \cdot B \cdot \Gamma\right| d t .
$$

The larger the foot radius is, the smaller the consumed energy is for the cyclic motion, even if the motion becomes unstable. Thus, the circular arc feet are effective in reducing the consumed energy.

\section{E. Optimal Radius}

There is a trade-off property between the convergence speed and the basin of attraction. What we can say is that the

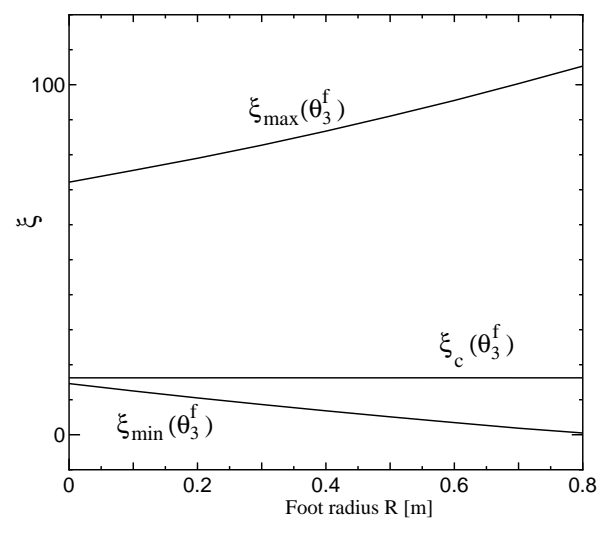

Fig. 11. Basin of attraction of $\xi$ w.r.t. the foot radii $R$. The area between the line of $\xi_{\min }\left(\theta_{3}^{f}\right)$ and $\xi_{\max }\left(\theta_{3}^{f}\right)$ is the basin of attraction by the numerical method. The line $\xi_{c}$ means the cyclic motions. In the upper area of $\xi_{\max }\left(\theta_{3}^{f}\right)$, vertical reaction forces are negative. There would be a flight phase. In the lower area of $\xi_{\min }\left(\theta_{3}^{f}\right)$, the velocity of the monotonic variable after the impact is not large enough to produce a step, $\xi_{\min }\left(\theta_{3}^{f}\right)$ is given by (44). After the beginning of the step, the biped goes backward or stands still eventually.

nearer the radius is to the leg length, the slower the speed of convergence is and the larger the basin is. 'Foot clearance problem' does not appear because of the variable length legs in our case. In the cases of 'Anthropomorphic Model' and 'Simplest Model' of Adamczyk's result [16], the CoM mechanical work property with respect to feet radii is similar to our result of consumed energy. However, in their cases of 'Forward-foot Model' and 'Kneed Model', the work had a minimum.

The suggestion of McGeer's to choose a foot radius of $1 / 3$ of leg lengths can also be considered in our discussion. It might be better to choose a larger radius (e.g. between a half and three quarters) to have a large basin of attraction even if the speed of convergence is worth.

\section{F. Unstable Walking with radii greater than the leg length}

Kuo's analysis [18] of the CoM velocity contradicts our study because he considers a simple model with rigid legs and circular arc feet and the CoM is located at hip position, and we consider prismatic knees. The lower part of Fig.6 presents the evolution of the CoM relative to the simple model of [18]. Tangent vectors of right ends of lines are expressing the preimpact velocity of CoM, and tangent vectors of left ends of lines are expressing the post-impact velocity of CoM. When $\mathrm{R}>0.8[\mathrm{~m}]$, the change of CoM velocities are upward, which means the impulsive force at the impact is negative. It actually would be a flight phase. Upper part of Fig.6 gives the CoM evolution in the case of our biped shown in Fig.2. Since all of the ranges of velocities of CoM at the impact are downward, it never fails to flight phase for any radius. In fact, our biped has prismatic knees and CoM is mainly distributed on the torso which is swinging a little. A lot of paths can be chosen for the CoM position differently from the simple model. 

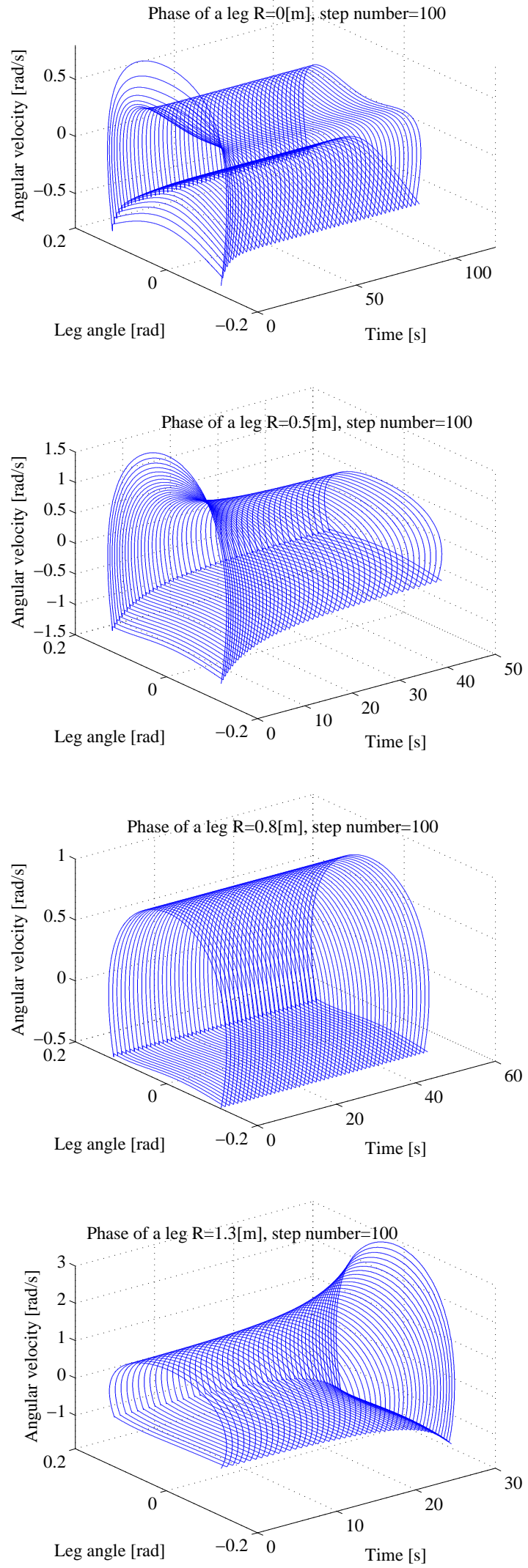

Fig. 12. Time evolutions of phases for the first leg at the foot radii $R=0$ [m] (stable), $0.5[\mathrm{~m}]$ (stable), $0.8[\mathrm{~m}]$ (neutral) and $1.3[\mathrm{~m}]$ (unstable) from the top.

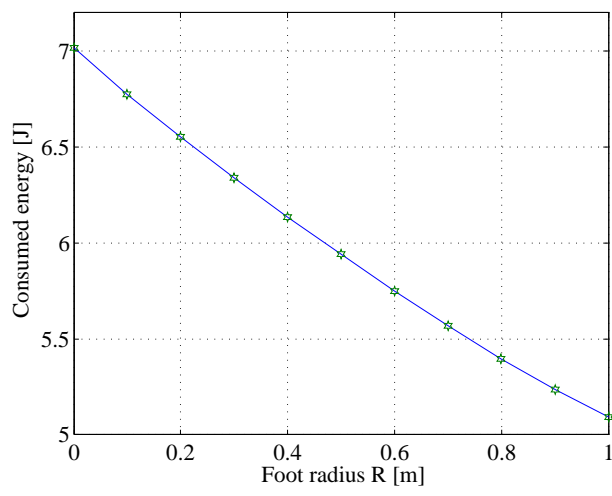

Fig. 13. Consumed energy for one cyclic step w.r.t. the foot radii $\mathrm{R}$ by the numerical simulation. The torso angle is chosen so that $\xi=16.27$ by the analytic solution for all $\mathrm{R}$. For $R=0.9[\mathrm{~m}]$ and $1.0[\mathrm{~m}]$, that is, unstable motions, the energy is computed for the cyclic motion.

\section{CONCLUSION}

In the paper, some effects of circular arc feet for a planar biped via a geometric tracking were taken into account. An analytic solution of Poincaré map was given for the controlled system. Stability of walking was analyzed by the Poincaré map and the following results are obtained:

- Radii of the circular arc feet affect the stability of walking, and the speed of convergence decreases when the radii approaches to a leg length.

- A basin of attraction is broadened by choosing larger radii and the controller can stabilize the biped walking in the largest basin of attraction for the radii less than the leg length.

The leg length and the radius smoothen the variation and reduce the impact velocity. For the same condition of the reference paths, the radius is significant for the stability and the basin of attraction. The results are analogous to those [11] [12] and the prospect [13] on passive dynamic walking. The geometric tracking method does not change the general effect of the circular arc feet. A reduction of the vertical CoM variation by the foot radius is functional not only for the geometric tracking method but for general biped walking. However the motion of CoM and the consumed energy are different from some very simple models because our model has variable length of legs and a torso.

\section{REFERENCES}

[1] Y. Aoustin, A. M. Formal'sky, "Design of Reference Trajectory to Stabilize Desired Nominal Cyclic Gait of a Biped", Proceedings of the International Workshop on Robot Motion and Control, ROMOCO'99, pp. 159-165, 1999.

[2] J. Grizzle, G. Abba and F. Plestan, "Asymptotically stable walking for biped robots: Analysis via systems with impulse effects", IEEE transaction on Automatic Control, vol. 46, no. 1, pp. 51-64, 2001.

[3] Y. Aoustin and A. M. Formal'sky, "Control Design for a Biped Reference Trajectory Based on Driven Angles as Functions of the Undriven Angle", Journal of Computer and System Sciences International, Vol. 42, No. 4, 159-176, 2003.

[4] C. Chevallereau, et. al., "Rabbit: A test bed for advanced control theory", IEEE Control System Magazine, Vol. 23, No. 5, pp. 57-78, 2003. 
[5] S. Kajita and K. Tani, "Study of Dynamic Biped Locomotion on Rugged Terrain - Derivation and Application of the Linear Inverted Pendulum Mode -", Proceedings of IEEE International Conference on Robotics and Automation, pp. 1405-1411, 1991. (in Japanese)

[6] E.R. Westervelt, G. Buche, and J.W. Grizzle, "Experimental Validation of a Framework for the Design of Controllers that Induce Stable Walking in Planar Bipeds", International Journal of Robotics Research, Vol. 24, No. 6, pp. 559-582, 2004.

[7] T. Kinugasa, et al. "Passive Walking of Biped Emu with Attitude Control of Body", Proceedings of IEEE/RSJ International Conference on Intelligent Robots and Systems 2003, pp. 346-359, 2003

[8] T. Kinugasa, K. Osuka and S. Miwa, "Biped Walking by Variations of Knee Lengths and Attitude Control of a Body and its Frequency Analysis", Journal of the Robotics Society of Japan, Vol. 25, No. 3, 2007 (in Japanese).

[9] C. Chevallereau and D. Djoudi, "Feet can improve the stability property of a control law for a walking robot", Proceedings of International conference on Robotics and Automation 2006, pp.1206-1212, 2006.

[10] A. H. Hansen, et. al., "Roll-over shapes of human locomotor systems: effects of walking speed", Clinical Biomechanics, Vol. 19, No. 4, pp. 407414, 2004

[11] T. McGeer, "Passive Dynamic Walking", International Journal of Robotics Research, Vol. 9, pp. 62-82, 1990

[12] M. Wisse and J. van Frankenhuyzen, "Design and construction of mike; a $2 \mathrm{~d}$ autonomous biped based on passive dynamic walking", Proceedings of Conference on Adaptive Motion of Animals and Machines, Paper No. WeP-I-1, 2003

[13] M. Wisse et. al., "How to Keep From Falling Forward: Elementary Swing Leg Action for Passive Dynamic Walkers", IEEE Transactions on robotics, Vol.21, No.2, pp.394-401, 2005.

[14] M. Wisse et. al., "Ankle Springs Instead of Arc-shaped Feet for Passive Dynamic Walkers", Proceedings of Humanoids 2006, pp.110-116, 2006.

[15] F. Asano and ZW Luo, "The Effect of Semicircular Feet on Energy Dissipation by Heel-strike in Dynamic Biped Locomotion", Proceedings of IEEE International Conference on Robotics and Automation 2007, pp.3976-3981, 2007.

[16] P. G. Adamczyk, S. H. Collins and A. D. Kuo, "The advantages of a rolling foot in human walking", The Journal of Experimental Biology 209, pp.3953-3963, 2006.

[17] B. Morris and J.W. Grizzle, "A Restricted Poincare Map for Determining Exponentially Stable Periodic Orbits in Systems with Impulse Effects: Application to Bipedal Robots", Proceedings of IEEE Conference on Decision and Control 2005, pp.4199-4206, 2005.

[18] A.D.Kuo, "Energetics of Actively Powered Locomotion Using the Simplest Walking Model", Journal of Biomechanical Engineering, Vol.124, pp.113-120, 2001.

[19] C. Chevallereau, A. Formal'sky et D. Djoudi, "Tracking of a Joint Path for the Walking of an Underactuated Biped", Robotica,pp. 15-28.volume 22, 2004.

[20] K.D.Farrell, C.Chevallereau and E.R.Westervelt, "Energetic Effects of Adding Springs at the Passive Ankles of a Walking Biped Robot", Proceedings of IEEE International Conference on Robotics and Automation, pp.3591-3596, 2007. 